\title{
Por qué viajan o no viajan las personas: Un estudio exploratorio
}

\section{Why do people travel or don't travel: An exploratory study}

\author{
HEREDIA-CRUZ, Miriam Guadalupe $\uparrow_{\dagger}^{\prime}$ ESPARZA-GARCIA, Irma Guadalupe*, CLARK-MENDIVIL, \\ Yesenia y NUÑEZ-RAMIREZ, Marco Alberto
}

Instituto Tecnológico de Sonora, Departamento de Ciencias Administrativas

ID $1^{\text {er }}$ Autor: Miriam Guadalupe Heredia-Cruz / ORC ID: 0000-0001-7109-2166, CVU CONACYT ID: 1014112

ID $1^{\mathrm{er}}$ Coautor: Irma Guadalupe Esparza-García / ORC ID: 0000-0002-3715-0761, CVU CONACYT ID: 287445

ID $2^{\text {do }}$ Coautor: Yesenia Clark-Mendívil / ORC ID: 0000-0003-2032-7433, Researcher ID Thomson: S-7878-2018, CVU CONACYT ID: 290473

ID $3^{\text {er }}$ Coautor: Marco Alberto, Núñez-Ramírez / ORC ID: 0000-0001-5825-4482, CVU CONACYT ID: 317551

DOI: $10.35429 /$ JTMS.2019.14.5.23.28

Recibido 10 de Enero 2019; Aceptado 30 Marzo, 2019

\section{Resumen}

El turismo social es una rama del turismo que busca que todas las personas tengan por igual la oportunidad de realizar un viaje, y de esta manera crear un desarrollo en la comunidad donde se lleva a cabo dichos programas e incrementar el turismo interno, y con ello cumplir el derecho universal a tener vacaciones. En México, no todos tienen acceso a hacer valer este derecho ya que el viajar se ha convertido en una actividad solo para un grupo de personas. Existe muy poca información en estudios sobre los motivos de viaje de los mexicanos, es por eso la importancia de identificar los motivos que llevan a las personas a viajar o lo que los limita a viajar. Esta investigación es cuantitativa, alcance exploratorio y descriptivo, en donde se estudiaron a 300 sujetos, utilizando la encuesta como método de recolección, la cual dio como resultado que las personas viajan para obtener nuevas experiencias, por recreación, para conocer nuevas culturas y No viajan por falta de tiempo y de dinero, datos importantes para la creación de nuevas ofertas turísticas que estén al alcance de todas las familias mexicanas, buscando la introducción del turismo social a México.

Turismo, Calidad de vida, Desarrollo

\begin{abstract}
Social tourism is a branch of tourism that seeks to ensure that all people have the opportunity to travel, and in this way create a development in the community where such programs are carried out as well as increase domestic tourism, and thereby fulfill the universal right to have vacations. In Mexico, not everyone has access to enforce this right since travel has become an activity only for a group of people. There is not enough information in studies about the Mexicans travel motivations, which is why it is important to identify the reasons that lead people to travel or what limits them to travel. This research is quantitative, exploratory and descriptive, where 300 subjects were studied, using the survey as a method of collection which resulted that most of the subjects travel to obtain new experiences, for recreation, to learn about new cultures and they do not travel due to lack of time and money, important data for the creation of new tourist offers that are available to all Mexican families, looking for the introduction of social tourism to Mexico.
\end{abstract}

Tourism, Quality of life, Development

Citación: HEREDIA-CRUZ, Miriam Guadalupe, ESPARZA-GARCIA, Irma Guadalupe, CLARK-MENDIVIL, Yesenia y NUÑEZ-RAMIREZ, Marco Alberto. Por qué viajan o no viajan las personas: Un estudio exploratorio. Revista Transdisciplinaria de Estudios Migratorios. 2019, 5-14: 23-28

\footnotetext{
*Correspondencia al Autor (correo electrónico: iesparza@itson.edu.mx)

$\dagger$ Investigador contribuyendo como primer autor.
} 


\section{Introducción}

Según la Organización Mundial del Turismo (OMT, 2019), durante el año 2018 se produjo en todo el mundo 1.400 millones de viajes internacionales lo que representa el $10 \%$ del producto interno bruto mundial generando $1 \mathrm{de}$ cada 10 empleos. Gómez y López (2002), consideran al turismo como un generador de producto y consumo, puesto que actualmente la cantidad de productos y la adquisición de ellos son realmente grandes a nivel internacional.

En México según los datos del Instituto Nacional de Estadística y Geografía ([INEGI], 2017) el turismo representa el $8.7 \%$ de participación del PIB turístico, de acuerdo a la Secretaría de Turismo ([SECTUR], 2018), durante enero del 2018 en México la llegada de turistas internaciones fue de 3.4 millones mostrando un incremento anual de $12.7 \%$ con respecto al año anterior, por otra parte el ingreso de divisas paso a ser a mil 941 millones de dólares, cantidad que representa un incremento del $0.7 \%$ en enero del 2017.

El turismo ha estado presente desde tiempos remotos, según Fernández y Fernández (2018), el motivo por el que las grandes civilizaciones comenzaron a viajar fue por el comercio, colonizar nuevos territorios, por la alimentación y la curiosidad de explorar nuevos lugares. Mientras que Gonzales (2004) aporta que anteriormente los viajes se realizaban con el fin de descubrir, explorar, conocer, migrar, esto sin una fecha determinada de regresar a su lugar de origen, y es según el Ministerio de Educación y formación profesional (2004) en el siglo XIX donde los viajes se convirtieron en viajar por interés de conocer los sitios turísticos.

De acuerdo a Jablonska, Jaremko y Timÿaғk (2016), el turismo social dio inicio en Francia en 1963, después de haber sido aprobada por la Organización Internacional del Trabajo (OIT), este consistía en un convenio sobre vacaciones pagadas, posteriormente en 1984 la Declaración Universal de los Derechos Humanos establece el derecho a vacaciones pagadas; Pastoriza (2008) menciona que después de la Segunda Guerra Mundial las vacaciones pagadas se convirtieron en un tema que predominó pues las personas buscan obtener este beneficio, haciendo que se volviera obligatorio en una gran cantidad de países europeos.
Acorde con Schenkel (2017) en Latinoamérica el turismo social entra como iniciativa a partir del año 1970, específicamente en México el modelo del turismo social es una táctica que en los últimos años se ha intentado implementar de acuerdo con el Centro de documentación turística ([CEDOC], 1992).

En el año 2019, el portal web Reportur (2019), informa que el presidente de México, Andrés Manuel López Obrador, presenta la estrategia nacional de Turismo 2019-2024 en donde presenta distintas propuestas entre las cuales se encuentra el programa Sonrisas para México, este programa consiste en probar el proyecto piloto de Turismo social.

Acorde con Muñiz (2001) el turismo social transciende más allá del propio beneficio individual y/o colectivo que genera el acceso al ocio turístico, puesto que contribuyen al mantenimiento de la estructura turística, especialmente en determinados destinos y épocas del año, favoreciendo la generación de rentas y empleos.

Actualmente existe muy poca información sobre estudios acerca de los motivos de viaje de los mexicanos, razón importante para llevar a cabo la presente investigación, ya que el objetivo de este artículo es identificar los principales motivos por las que viajan o no viajan las personas a través de la aplicación de un instrumento con el fin de contribuir a la propuesta del turismo social en México en beneficio de la sociedad.

A continuación de la introducción se presenta el segundo apartado el cual consiste en la revisión de la literatura, para después seguir con el apartado de la metodología utilizada; y continuar con el cuarto apartado en donde se describen los resultados de la investigación. Para finalizar se puede encuentran los agradecimientos, las conclusiones y las referencias bibliográficas utilizadas.

\section{Revisión de la literatura}

Según la OMT (1994, citado por Sancho 1998), "el turismo comprende las actividades que realizan las personas durante sus viajes y estancias en lugares distintos a su entorno habitual, por un periodo de tiempo consecutivo inferior a un año, con fines de ocio, por negocios y otros" (p.11). 
Para SECTUR (2015), el turismo es una de las actividades que se realizan en un lugar diferente a tu entorno habitual, por un período de tiempo consecutivo inferior a un año, con fines de ocio. El concepto de turismo social es definido de muchas manera, de acuerdo con AllParty Parliamentary Group on Social Tourism (2011 citan a Family Holiday Association (FHA), el cual define al turismo social como la inclusión a las vacaciones a personas de bajos recursos económicos.

El motivo de viaje de las personas se puede describir a través de las variables según Álvarez (2018), como el género, la edad, el hábitat, el nivel de estudios, la ocupación. En la tabla que se presenta a continuación se puede observar los estudios empíricos que han investigado diferentes autores con el fin de identificar los motivos por los que viajan las personas, haciendo hincapié en que cada uno tiene diferentes muestra, método.

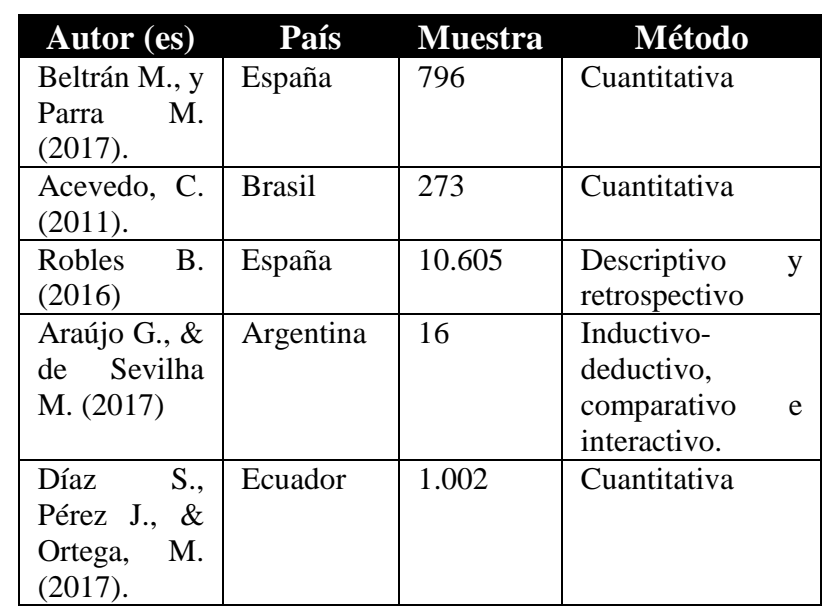

Tabla 1 Estudios empíricos sobre los motivos de viajes Fuente: Elaboración propia en base a los autores presentados en la tabla

\section{Metodología}

El método utilizado es un estudio de alcance cuantitativo, no experimental, exploratorio y descriptivo y de corte transaccional, según Landeau (2007) es tipo cuantitativo ya que mide conceptos o variables, según Hernández, Fernández y Baptista (2006), es no experimental ya que no se manipularon los sujetos de estudio, es exploratoria porque se está examinando un tema poco estudiado, descriptivo debido a que se caracteriza el fenómeno de estudio y transversal porque se realizó en un periodo de tiempo, así como también la evaluación del universo para identificar propiedades importantes que permitan informar sobre el fenómeno estudiado.
El total de los sujetos fue de 300 , a los cuales se les aplico un cuestionario de gradación Likert-10. Como se puede observar en la tabla 2, se presentan las características sociodemográficas.

Características de los sujetos participantes en el estudio $(\mathrm{N}=300)$.

\begin{tabular}{|c|c|c|}
\hline \multicolumn{3}{|l|}{ Genero } \\
\hline Femenino & 181 & 60.3 \\
\hline Masculino & 119 & 39.7 \\
\hline \multicolumn{3}{|l|}{ Edad } \\
\hline 18 a 30 años & 210 & 70.1 \\
\hline 31 a 50 años & 60 & 20.1 \\
\hline 51 a 70 años & 30 & 9.9 \\
\hline \multicolumn{3}{|l|}{ Estado Civil } \\
\hline Soltero & 211 & 70.3 \\
\hline Casado & 76 & 25.5 \\
\hline Otro & 13 & 4.3 \\
\hline \multicolumn{3}{|l|}{ Grado académico } \\
\hline Secundaria & 3 & 1.0 \\
\hline Técnico & 92 & 30.7 \\
\hline Licenciatura & 149 & 49.7 \\
\hline Maestría & 49 & 16.3 \\
\hline Doctorado & 7 & 2.3 \\
\hline \multicolumn{3}{|l|}{ cupación } \\
\hline Estudiante & 94 & 31.3 \\
\hline Empleado & 142 & 47.3 \\
\hline Profesionista/Técnico & 36 & 12 \\
\hline Ama de casa & 1 & .5 \\
\hline Directivo/ejecutivo & 9 & 3.0 \\
\hline Dueño de un negocio & 9 & 3.0 \\
\hline Retirado & 9 & 3.0 \\
\hline \multicolumn{3}{|l|}{ Ingreso económico } \\
\hline$\$ 5000$ o menos & 65 & 21.7 \\
\hline$\$ 6000$ a 10000 & 88 & 29.3 \\
\hline$\$ 11000$ a 15000 & 47 & 15.7 \\
\hline$\$ 16000$ a 20000 & 41 & 13.7 \\
\hline$\$ 21000$ a 250000 & 26 & 8.7 \\
\hline \$26000 o más & 33 & 11 \\
\hline
\end{tabular}

Tabla 2 Caracterización de los sujetos de estudio Fuente: Elaboración Propia

El cuestionario utilizado como método de recolección de datos se conformó de 3 secciones, la sección I contribuye a identificar las características sociodemograficas de la muestra, también se encuentran unas preguntas filtro para identificar quién ha realizado o no un viaje, la sección II se conforma de 20 ítems el cual busca identificar los motivos por el cual viajan las personas y la sección 3 se conforma de 9 ítems para identificar el motivo por el cual no realizan al menos un viaje las personas. 
La Escala utilizada fue de tipo Likert de 10 puntos para medir los niveles de acuerdo/desacuerdo con respecto a las variables de motivación cuyos cálculos tenían el rango entre $1=$ Totalmente en desacuerdo y el $10=$ Totalmente de acuerdo. A continuación se presenta la descripción en la tabla 3:

\begin{tabular}{|l|l|l|}
\hline Dimensiones & \multicolumn{1}{l}{ Ítems } & \multicolumn{1}{l|}{ Alfa de Cronbach } \\
\hline Personas que no viajan & 20 & .816 \\
\hline Personas que sí viajan & 9 & .768 \\
\hline
\end{tabular}

Tabla 3 Operacionalizacion de las variables Fuente: Elaboración Propia

\section{Resultados}

Los principales resultados encontrados en esta investigación se darán a conocer por un lado describiendo las características de los sujetos en estudio de acuerdo a los datos sociodemográficos siendo los siguientes; el $60.3 \%$ de los sujetos son género femenino y el $39.7 \%$ género masculino, soltero con un $70.3 \%$ y el $25.3 \%$ casado con una edad promedio de 18 30 años con un $70.1 \%$, la mayoría es empleado con un $47.3 \%$, Cabe mencionar que los rangos económicos de los sujetos tienen un equilibrio de los porcentajes con relación a los rangos definidos representando menos a $\$ 5,000$ el $21.7 \%$, de $\$ 6,000$ a $\$ 10,000$ con un 29.3 , de $\$ 11,000$ a $\$ 20,000$ con un $29.4 \%$, con ingreso económico mayor a $\$ 26,000$ con el $11 \%$.

De acuerdo a la muestra las personas respondieron en un $76.3 \%$ que si han realizado un viaje y con un $23.7 \%$ que no ha realizado un viaje en los últimos 3 años como se puede visualizar en la figura 1.

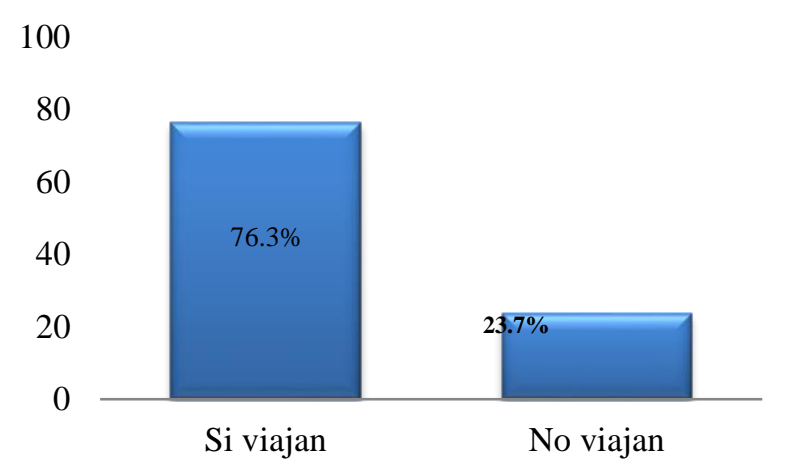

Figura 1 Participantes del estudio que si viajan o no viajan Fuente: Elaboración Propia
Por otro lado a continuación se presentaron los resultados de acuerdo a los estadísticos descriptivos generados en el SPSS, a través de la tabla 4 , donde se puede identificar las principales razones por las cuales los sujetos han realizado un viaje, siendo las siguientes: 1) por tener nuevas experiencias, 2) Conocer nuevas culturas porque cuentan con estabilidad económica, 3) porque cuentan con estabilidad económica, 4) por conocer diferentes lugares, 5) por recreación, 6) para pasar tiempo con la familia y 7) para aprender. Por otro lado cabe resaltar que el contar con estabilidad económica permite a las personas realizar los viajes y en la tabla 5 se puede observar que el motivo principal por el que no viajan las personas es por falta de tiempo y de dinero, no es el miedo a viajar o gastar sino más bien que el vivir al día llegan a un punto de considerar que viajar no es una prioridad.

\begin{tabular}{|c|c|c|c|c|c|}
\hline & $\mathrm{N}$ & Mínimo & Máximo & Media & $\begin{array}{l}\text { Desv. } \\
\text { típ. }\end{array}$ \\
\hline $\begin{array}{l}\text { 1. Porque cuento } \\
\text { con estabilidad } \\
\text { económica }\end{array}$ & 231 & 1 & 10 & 7.42 & 2.394 \\
\hline $\begin{array}{l}\text { 2. Porque puedo } \\
\text { pagar vacaciones }\end{array}$ & 231 & 1 & 10 & 7.17 & 2.508 \\
\hline $\begin{array}{l}\text { 3. Por cumplir mis } \\
\text { gustos y lujos }\end{array}$ & 231 & 1 & 10 & 6.71 & 2.820 \\
\hline $\begin{array}{l}\text { 4. Por conocer } \\
\text { diferentes lugares }\end{array}$ & 231 & 1 & 10 & 7.97 & 2.696 \\
\hline $\begin{array}{l}\text { 5. Para olvidarme } \\
\text { de problemas }\end{array}$ & 231 & 1 & 10 & 5.74 & 3.361 \\
\hline $\begin{array}{l}\text { 6. Salir de la zona } \\
\text { de confort }\end{array}$ & 231 & 1 & 10 & 6.82 & 3.022 \\
\hline $\begin{array}{l}\text { 7. Sacar el estrés } \\
\text { de la vida diaria }\end{array}$ & 231 & 1 & 10 & 7.23 & 3.046 \\
\hline 8. Por recreación & 231 & 1 & 10 & 7.74 & 2.772 \\
\hline 9. Por salud & 231 & 1 & 10 & 5.79 & 3.288 \\
\hline $\begin{array}{l}\text { 10. Por pasar } \\
\text { tiempo con la } \\
\text { familia }\end{array}$ & 231 & 1 & 10 & 7.60 & 3.057 \\
\hline $\begin{array}{l}\text { 11. Tener nuevas } \\
\text { experiencias }\end{array}$ & 231 & 1 & 10 & 8.48 & 2.484 \\
\hline 12. Para aprender & 231 & 1 & 10 & 7.80 & 2.820 \\
\hline $\begin{array}{l}\text { 13. Para conocer } \\
\text { culturas }\end{array}$ & 231 & 1 & 110 & 8.35 & 7.275 \\
\hline $\begin{array}{lr}14 . & \text { Para } \\
\text { encontrarse } & \text { con } \\
\text { uno mismo } & \\
\end{array}$ & 231 & 1 & 10 & 6.16 & 3.351 \\
\hline $\begin{array}{l}\text { 15. Por eventos } \\
\text { religiosos }\end{array}$ & 231 & 1 & 10 & 2.99 & 2.970 \\
\hline $\begin{array}{ll}16 . \quad \text { Visita } & \text { a } \\
\text { familiares } & \text { o } \\
\text { amigos } & \end{array}$ & 231 & 1 & 10 & 6.55 & 3.584 \\
\hline $\begin{array}{lr}\text { 17. Porque } & \text { tengo } \\
\text { la cultura } & \text { de } \\
\text { ahorrar } & \text { para } \\
\text { vacaciones } & \end{array}$ & 231 & 1 & 10 & 6.08 & 3.457 \\
\hline $\begin{array}{l}\text { 18. Por pertenecer } \\
\text { a un grupo }\end{array}$ & 231 & 1 & 10 & 2.80 & 2.668 \\
\hline $\begin{array}{l}\text { 19. Por sentirme } \\
\text { aceptado }\end{array}$ & 231 & 1 & 10 & 2.67 & 2.736 \\
\hline 20. Por negocios & 231 & 1 & 10 & 3.32 & 3.318 \\
\hline
\end{tabular}

Tabla 4 Estadísticos descriptivos de los sujetos que sí viajan

Fuente: Elaboración propia basada en SPSS 


\begin{tabular}{|c|c|c|c|c|c|}
\hline & $\mathrm{N}$ & Mínimo & Máximo & Media & $\begin{array}{l}\text { Desv. } \\
\text { típ. }\end{array}$ \\
\hline $\begin{array}{l}\text { 1. Por no contar con } \\
\text { estabilidad } \\
\text { económica }\end{array}$ & 69 & 1 & 10 & 5.96 & 3.521 \\
\hline $\begin{array}{l}\text { 2. Por cuestiones de } \\
\text { trabajo }\end{array}$ & 69 & 1 & 10 & 5.96 & 3.516 \\
\hline $\begin{array}{l}\text { 3. Por falta de } \\
\text { tiempo y dinero }\end{array}$ & 69 & 1 & 10 & 6.77 & 3.227 \\
\hline $\begin{array}{l}\text { 4. Por razones de } \\
\text { seguridad } \\
\text { económica }\end{array}$ & 69 & 1 & 10 & 5.38 & 3.465 \\
\hline 5. Por miedo & 69 & 1 & 10 & 2.68 & 2.512 \\
\hline 6. Por no gastar & 69 & 1 & 10 & 4.67 & 3.563 \\
\hline $\begin{array}{l}\text { 7. Porque no me } \\
\text { comprometo } \\
\text { ahorrar para un } \\
\text { viaje }\end{array}$ & 69 & 1 & 10 & 4.90 & 3.667 \\
\hline $\begin{array}{l}\text { S2.8 Porque no es } \\
\text { una prioridad viajar }\end{array}$ & 69 & 1 & 10 & 5.29 & 3.308 \\
\hline $\begin{array}{l}\text { S2.9 Por falta de } \\
\text { oportunidades en el } \\
\text { campo laboral }\end{array}$ & 68 & 1 & 10 & 4.66 & 3.531 \\
\hline
\end{tabular}

Tabla 5 Estadísticos descriptivos de los sujetos que no viajan

Fuente: Elaboración propia basada en SPSS

\section{Agradecimiento}

Publicación apoyada por el Programa de Fomento y Apoyo a la Investigación (PROFAPI) 2019, a través del Instituto Tecnológico de Sonora, en Ciudad Obregón, Sonora, México

\section{Conclusiones}

De acuerdo a la investigación realizada sobre las razones por las cuales viajan o no viajan las personas se puede decir que se cumplió con el objetivo ya que se lograron identificar esas razones.

Los resultados arrojados fueron muy significativos ya que permitieron realizar una serie de análisis, en donde se pudo identificar que la razón por lo que viajan las personas es para tener nuevas experiencias, conocer nuevas culturas, entre otras variables, ya que cuentan con una estabilidad económica que se los permite, mientras que el motivo por el cual no realizan un viaje es debido a la falta de tiempo y dinero, o bien al no tener una cultura de tomar vacaciones consideran que el viajar no es una prioridad.

La presente investigación puede dar lugar a nuevas investigaciones referente a los motivos de viaje puesto que se considera importante el fomentar la realización de este tipo de estudios en México, debido a que existen muy pocos relacionados a este tema.
Por otro lado como recomendación metodológica dado que la muestra no presenta un muestreo probabilístico para futuras investigaciones se sugiere emplear un muestreo estratificado para dividir a la población en subgrupos y seleccionar aleatoriamente a los sujetos finales de los diferentes estratos en forma proporcional y obtener conclusiones que permita la creación de programas de viaje para diferentes mercados.

De esta manera se concluye que el turismo social presenta una oportunidad para hacer cumplir el derecho universal de vacaciones, a través de los programas que apoyan este tipo de turismo se fomentará en las personas el gusto por la recreación y el disfrute del tiempo libre, aportando al turismo interno y al desarrollo económico en el lugar donde se presente.

\section{Referencias}

Acevedo, C. (2011). Motivos para viajar: Un estudio con turistas maduros en el contexto brasileño. FACEF Pesquisa-Desenvolvimento e Gestão, 6(3).

All-Party Parliamentary Group on Social Tourism (2011) Investigación sobre los beneficios sociales y económicos del turismo social. Recuperado de: http://d3n8a8pro7vhmx.cloudfront.net/appgons ocialtourism/pages/29/attachments/original/111 031GivingBritainaBreak.pdf?1320318284

Álvarez, A. (2018) Estado de bienestar y turismo para todos. Motivos para no viajar. Cuadernos De Turismo,

https://doi.org/10.6018/turismo.41.326941

Araújo G., y Sevilha M. (2017) Los viajeros y sus motivaciones un estudio exploratorio sobre quienes aman viajar. Recuperado de: http://www.redalyc.org/pdf/1807/18074918200 4.pdf

Beltrán M., y Parra M. (2017). Perfiles turísticos en función de las motivaciones para viajar. Cuadernos de Turismo, (39), 41-65.

CEDOC (1992) Seminario de animación turística México-Francia. México: SECTUR \& CESTUR. 
Díaz S., Pérez J., \& Ortega, M. (2017) Análisis de las motivaciones hacia el ecoturismo en Ecuador. Un estudio de caso en la Isla Santay. Revista Turydes: Turismo y Desarrollo, 10(22), 1-18.

Fernández A., y Fernández J. (2018) Paisajes y turismo. UNED: Madrid

Gómez B., y López F. (2002) Regionalización turística del mundo. España: Ediciones Universidad de Barcelona.

González D. (2004) El anfitrión como actor social en el turismo. Reflexiones desde el caso de Ixtapan de la Sal, México. Revista de Ciencias Sociales (CR), 3(105).

Hernández R., Fernández C., y Baptista P. (2006) Metodología de la investigación. McGraw-Hill: México

INEGI (2017) Turismo. Producto Interno Bruno turístico. Recuperado de: https://www.inegi.org.mx/temas/turismosat/

Jablonska J., Jaremko J. y Timÿaғk M. (2016). Turismo social, clientes y perspectivas., Vol. 7 No. 3. DOI: $10.5901 / \mathrm{mjss} .2016 . v 7 \mathrm{n} 3 \mathrm{~s} 1 \mathrm{p} 42$

Landeau, R. (2007) Elaboración de trabajos de investigación. Alfa: Venezuela

Ministerio de Educación y formación profesional (2004) Servicios socioculturales: La cultura del ocio. Secretaría general técnica.

Muñiz (2001) La política de turismo social. Consejería de Turismo y Deporte, Dirección General de Planificación Turística. Sevilla.

OMT (2019) Las llegadas de turistas internacionales suman 1.400 millones dos años antes de las previsiones. Recuperado de: http://www2.unwto.org/es/press-release/2019-

01-21/las-llegadas-de-turistas-internacionalessuman-1400-millones-dos-anos-antes

Pastoriza, E. (2008) El turismo social en la Argentina durante el primer peronismo. Mar del Plata, la conquista de las vacaciones y los nuevos rituales obreros, 1943-1955. Mundo Mundos Nuevos.
Reportur. (2019) AMLO: Estrategia de turismo 2019-2024 tendrá 5 ejes. Recuperado de: https://www.reportur.com/mexico/2019/02/25/1 opez-obrador-presenta-estrategia-nacionalturismo-2019-2024-basada-5-ejes/

Sancho A. (1998) Introducción al turismo. Publicaciones de la OMT en educación turística, Organización Mundial del Turismo.

SECTUR (2018) Resultados de la Actividad Turística Enero 2018. Recuperado de: https://www.datatur.sectur.gob.mx/RAT/RAT2018-01(ES).pdf

SECTUR. (2015) Portal del Sistema Nacional de Información Estadística y Geográfica de Turismo - SNIEGT. Glosario. Recuperado de: http://www.datatur.sectur.gob.mx/SitePages/Gl osario.aspx\#Glosario_T

Schenkel E. (2017) Turismo social en América latina: la conquista de un derecho desigual. Recuperado de: http://www.ingeba.org/lurralde/lurranet/lur41/4 1erica.pdf 